\title{
The Science of Group and Organizational Processes
}

\author{
Kenneth D. Mackenzie ${ }^{1,2}$ \\ ${ }^{1}$ School of Business, University of Kansas, Lawrence, KS 66045, USA \\ ${ }^{2}$ EMAC Assessments, LLC, 1555 Fountain Drive, Lawrence, KS 66047, USA \\ Correspondence: Kenneth D. Mackenzie, EMAC Assessments, LLC, 1555 Fountain Drive, Lawrence, KS 66047, \\ USA. Tel: 1-785-749-1006. E-mail: kdmackenzie@sunflower.com
}

Received: August 30, 2012 Accepted: October 10, 2012 Online Published: October 26, 2012

doi:10.5539/emr.v1n2p133 URL: http://dx.doi.org/10.5539/emr.v1n2p133

\begin{abstract}
This article is the first of a pair about the representation of group and organizational processes. The article begins with a model of the science of group and organizational processes. It depicts the main subcommunities and how their interdependence affects their progress. This interdependence would be improved if there was a common definition and representation for group and organizational processes. Recognizing that there are many existing concepts of group and organizational processes and several efforts to create a representation, it is clear that more work is required. A common representation of group and organizational processes ought to meet five criteria in order to be feasible. Then, to select among the feasible candidates, a set of ten desiderata for comparing, contrasting, and synthesizing them is presented.
\end{abstract}

The second article in this series derives a working common representation of group and organizational processes.

Keywords: consulting, group process, organizational process, process, representation, technology, science

\section{A Model of Science}

Science can be viewed in terms of interdependent communities of persons with a shared interest and commitment to the evolution of a body of knowledge. Science is more than the knowledge it has produced because, hopefully, this knowledge is improving. Nor can science be defined solely in terms of its methods because these methods change. It is well known that some sciences exhibit greater rates of progress than others. For example, the science of molecular biology since the discovery of the structure of DNA by Crick and Watson (1953) has led to rapid and profound changes in the basic understanding of biology. At about the same time, some early organizational theorists started to think about organizational learning. This science of organizational learning has not made as much progress (Mackenzie, 1994). Overall, the biological and physical sciences exhibit more rapid development than do the many social sciences. Why?

Why do some sciences progress more rapidly than others? Consider the science of navigation. Prior to Nicolaus Copernicus, astronomy was based on the geocentric idea that the heavens revolved the earth. Copernicus proposed the heliocentric theory that the earth, in fact, revolved around the sun. At that time, astronomy was encrusted with conflicting theological determinants which could not be tested empirically. It lacked flexible engineering to convert the astronomical theories into useful application. There were inadequate tools for estimating distances and time, and astronomy was applied mostly for seasonal prediction, primitive navigation, and the timing of ceremonial celebrations. Then came the invention of the telescope by Galileo Galilei, which enabled Galileo to begin looking more closely at celestial bodies. He made important discoveries that stimulated others to use telescopes which led to more discoveries. These observations required greater precision about the measurement of distance and time and many technological advances occurred. Increasingly, because there was a growing community of astronomers using telescopes, common measurements of distance and time became vital in comparing observations. Technological developments yielded increasingly precise information about the heavens which led, in turn, to advances in theory. Greater knowledge of astronomy stimulated improvements in applications to improve navigation methods and allowed the Netherlands to become a major trading nation. The history of astronomy has been the result of the interplay of improved theory, better tools, more development of engineering methods to apply the tools and theories, rapid improvements in the methods of navigation, and to better and safer shipping. The functional interdependence among the theorists, the engineers, and the tool developers, and the appliers continues today in both deep water exploration and in space. 
Let us propose that scientific communities include at least four subcommunities: the theorists, the engineers, the tool makers, and the appliers. Developments in any of the subcommunities may spark developments in the others in an endless round of interdependent advancements. It should be noted that developments within a subcommunity can be made independently of those of another. Indeed, advances in a subcommunity are not always done solely for the benefit of another. For example, new computing tools benefit many scientific and technological subcommunities.

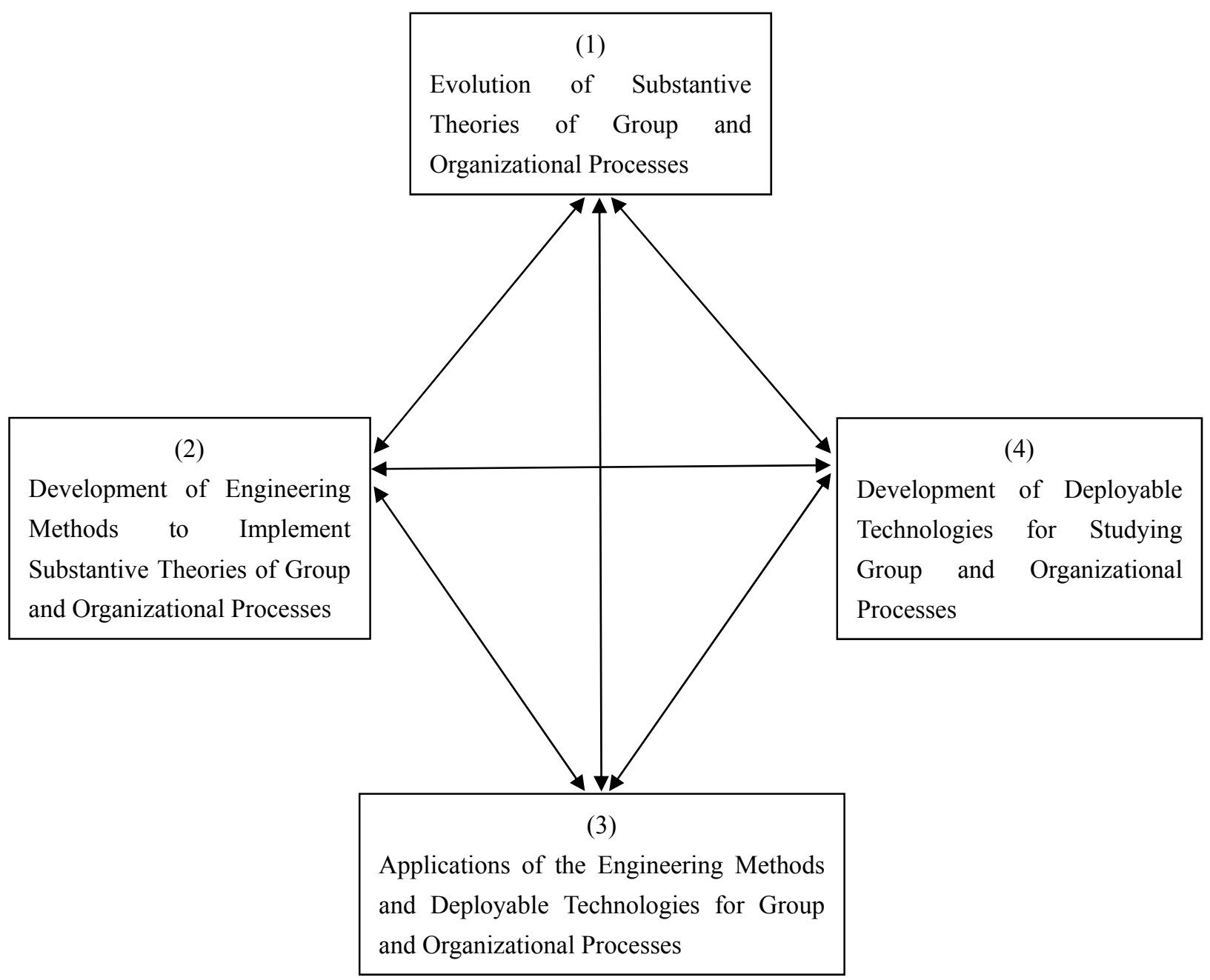

Figure 1. The science of group and organizational processes

Now consider the science of group and organizational processes. Taken as a whole it is not totally unfair to conclude that this scientific field is still in its formative stages. For example, there is no agreement on the very concept of a process. There is consequently no agreement about the need to represent group and organizational processes, let alone how to do so. However, the subcommunities exist. They are just not well developed and for the most part, their interdependence is relatively low. There are lots of substantive theorists and existing theories for group and organizational processes. These include, but are not limited to, the analysis of organizational learning, leadership, and decision making, etc. Tools have been developed for representing the technical aspects of processes in the form of computer models, universal modeling languages, artificial intelligence, and mathematical statistics. Melcher (2012) has evolved a research approach and analyses of organizational behavior using dynamic systems analysis. Notable efforts to engineer group and organizational processes include organizational development, business process management, quality assurance, organizational design, and managing order-entry cycles and logistics. There are a few conspicuous exceptions to the lack of engineering such as the MIT Process Dictionary (Malone, Crowston, and Hermann, 2003), many computer tools to formulate and manage execution business processes, the pioneering analytical work by Sowa (2000), and organizational 
design (cf. Mackenzie, 1986 and Kilmann, 2001). But for the most part, the group and organizational sciences lack the robust interplay among their subcommunities. The science of group and organizational processes will be more productive when developments in its subcommunities advance and the interdependencies among them are enhanced.

A model for the science of group and organizational processes is depicted in Figure 1. Box 1 represents the subcommunity of those developing and testing substantive theories of group and organizational processes. This subcommunity is large and industrious, as evidenced by the world-wide proliferation of journals, books, etc. Box 2 is the subcommunity of those seeking to invent better methods for engineering the implementation of substantive theories. One area in the engineering that shows promise is the field of organizational design and development (cf. Kilmann, 2001, and Mackenzie, 1986). Box 3 depicts the subcommunity involved in the application of these engineering methods and deployable technologies. The members of this subcommunity are primarily the management and staff within organizations seeking to improve the delivery of goods and services, and consulting firms. The fourth box is the subcommunity whose interests are in the development of improved deployable technologies for the study and analysis of group and organizational processes. This subcommunity consists of information technologists, mathematical statisticians, and those who work on modeling group and organizational processes (GOPs).

The six arrows connecting these four subcommunities of the series of group and organizational processes represent the interdependence among the subcommunities. These interdependencies are still relatively sparse but growing within the scientific community for the science of group and organizational processes.

The main thesis of this article is that there will be more rapid development in the science of group and organizational processes when these subcommunities exhibit greater interdependence. That way developments in any one subcommunity will spark developments in the others. The second thesis of this article is the need to agree on a definition of a group and organizational process and its representation, which is the intellectual precursor to developing a common representation of GOPs. The third thesis is that a common concept and representation of group and organizational processes (GOPs) provides the basis for improving the ability, capacity, and the need for its four subcommunities to work together more productively.

\section{Processes and Group and Organizational Behavior}

Processes are intuitive and natural in the organization sciences because individual, group, and organizational behavior is processual. Consequently, the study of GOPs ought to explicitly incorporate processual thinking. This is not a new idea. For example, Pettigrew et al (2001) argue that processual thinking should be central to the study of change and organizational development. Furthermore, Pettigrew also (1985) believes that it is faulty to view organizational change without considering the context, the history, and the processes involved. Others, such as Greenwood and Hinings (1996), Orlikowski (1996), and Mackenzie (2000, 2004) concur. Static studies of organizational change which ignore time are considered deficient by Pettigrew (1997) and Van de Ven et al. (1989), among others.

The use of longitudinal investigations for the study of organizational change was applied by Pettigrew and Whipp (1991). Andrew Van de Ven and colleagues (cf. Van de Ven, et al., 1989; Van de Ven \& Huber, 1990) pioneered methods for studying processes of organizational change. Karl Weick (1969) argued that one should focus more on the processes of organizing than on the entity called the organization, and before that Cyert and March (1963) stressed the advantages of examining organizational decision-making processes. Mackenzie (1976 a, b) developed process models for analyzing small group structural change in little laboratory groups and illustrated them by analyzing group behavior in a ten year series of laboratory experiments. Later, Mackenzie (1986) proposed and tested explicit models for designing organizations and illustrated them with the design of a regional supermarket organization.

There are many interconnected analytical challenges in studying organizational change. These include: (1) dealing with multiple contexts and multiple levels of analysis; (2) incorporating time, history, processes, and action; and (3) formulating notions of change processes and their linkages to organizational performance outcomes. While these processual challenges are inherently sensible, basic, and central to the organizational sciences, the publication of explicit and mathematical representations of processual theories is still a tiny fraction of the literature. This is a curious omission for a scientific field as extensive as the organization sciences.

A relatively complete description and analysis of actual GOPs cannot be done adequately in a short journal article. Pettigrew (1997) argues that one reason is that short articles make it hard to show both scholarly quality and practical relevance. The lack of consensus on the nature of the representation of GOPs means that a large portion of an article requires extensive development which is limited by page constraints. Avital (2000) asked researchers why they limited themselves to static "snap shot" research. An answer provided by Avital is that it is simply easier to publish this type of research. A rigorous foundation must rest on a shared and agreed upon 
formulation of GOP representation. A common GOP representation would enable authors to shorten their articles.

Describing GOPs is labor intensive and conceptually challenging. Each case study can only describe a limited set of GOPs. Absent a common definition and representation of GOPs, the cumulation of results is restricted and this hinders generalization of findings. Over time, it is expected that these empirical processual studies will eventually result in scientific breakthroughs and will encourage the subcommunities to interact to produce useful results. Despite this promise, most publications end up reducing the richness of GOPs to variables rather than dealing with the GOPs themselves. Converting richer descriptions of GOPs into encapsulating variables remains the commonly used method for stating and testing theories about group and organizational behavior. Problems with this strategy have been noted by many scholars. For example, Mohr (1982), Numagami (1998) and Mackenzie (2007) argue that the variance approach may be an intellectual dead-end for discovery. So, why is the study of organizational phenomena in terms of actual GOPs not more central to the organizational sciences? At least part of the answer is the lack of a credible way of representing the varieties of GOPs. Another part is the relatively low levels of interaction among the subcommunities of the science of GOPs.

However, despite its obvious advantages, establishing a common definition and representation of GOPs remains problematic for many reasons: (1) GOPs can be described at different levels of detail; (2) the GOP in question can be affected by technology; (3) GOPs can both influence, and be influenced by others; and (4) a GOP is never an "orphan," as there is always another linking event, person, or process to which it is related. Should these linkages be included in the representation of a GOP? Furthermore, (5) there is the question of which GOP to represent and how one chooses those to incorporate into a study. (6) Should outcomes of a GOP be part of the GOP representation? (7) GOPs have consequences. Should the evaluation of the outcomes be included in its representation? (8) GOPs involve interdependence but how does one define interdependence?

These eight problems have academic and practical interest. For example, there are extensive business process management (BPM) tools which allow the creation of process frameworks supplemented by special features such as assigning priorities and personnel, specifying inputs and outputs, evaluating performance, making changes to integrate with other process frameworks, and even stimulating performance. These many features remind one of a powerful vacuum cleaner with many attachments that are designed for special cleaning jobs. They illustrate how the potential of a GOP representation can be adapted to include specific answers to practical applications. The power of existing BPM computer tools improves their applicability, but this very power obscures the features of the basic vacuum cleaner. The proposed common representation of GOP in the second article is analogous to the simple no-frills vacuum cleaner whose basic ontological properties are defined and developed.

There are also serious issues in the teleology of how to conduct process research, such as how to handle temporal issues, defining goals, selecting ones research methods and data collecting strategies and drawing inferences from the data. For example, A. Langley (1999) discusses seven strategies for theorizing from process data, such as composing one's narrative, quantification of incidents, using alternative theoretical templates, grounded theorizing, visual mapping, temporal decomposition, and making comparisons. Langley (2009) raises the important issue of how to deal with on-site relationships between the researcher and the host group or organization. There are ethical issues in making interventions in actual groups and organizations that cannot be ignored.

\subsection{Why Bother Representing Group and Organizational Processes?}

GOPs can be complex, dynamic, and subject to change. Given these issues and puzzles, is there a need for developing a common representation? We humans have managed to operate groups and organizations since the beginning of time without a formal representation of processes. So, why bother? The need for an answer may be compelling to some, while others may have this insurmountable pragmatic objection: properly representing GOPs may be more work than it is worth.

Here are ten arguments for developing methods for representing GOPs. (1) Some GOPs, such as quality assurance, business process reengineering, project management, etc., benefit by enhancing our ability to analyze, compare, and improve them. They seem to be worth the investments required. (2) A common representation of GOPs may help us avoid tautological explanations and irrefutable theories by emphasizing how behavior is occurring rather than by depending upon teleological arguments based on probable intentions. (3) GOPs are philosophically necessary because they are ontologically robust. An overall argument and history for this is provided by Rescher (1996). This ancient argument goes back to Heraclitus of Ephesus in the six century BCE and continued in the works of philosophers as varied as Plato, Aristotle, Leibniz, Hegel, Pierce, James, Bergson, Dewey, Whitehead, Sheldon, Heidegger, and more recently by Bogost (2006), and Harmon (2009). (4) It is logical to seek a means for representing GOPs in order to study them. (5) The use of variables instead of GOPs 
has yielded meager fruits, as demonstrated by Mohr (1982) and argued by MacIntyre (1984). (6) The variety of process research (cf. A. Langley, 2009) provides hope for new and fruitful advances by replacing the variance approach with a common representation of GOPs. (7) To some, it is fun and creative to formulate GOP behavior in terms of their processes. The argument for having fun and enjoyment in doing science is a powerful motivator. There are three other technical reasons (cf. Mackenzie 2006, 2009) which rely on the GOP representation called process frameworks. (8) Process frameworks can be reduced to their process skeletons and process skeletons can be converted into analytical functions. (9) Process frameworks are useful for multi-level GOPs. (10) Process skeletons provide an improved ability to conduct and test theories and models of GOPs.

\subsection{Is it Worth While to Try and Construct a Solution?}

The search for a common representation of a class of related phenomenon has proven valuable in the evolution of alchemy to chemistry, and in other sciences, such as biology. Biology has gone from classification of species by their outward physical appearance to their DNA and functional relationships among genes. Finding a common representation of GOPs might have comparable advantages for the science of group and organizational processes. A processual approach offers substantial advantages over testing group and organizational behavior by variables. How can an organizational theory ever be tested when its constituent GOPs are inaccurately represented and become encapsulated as variables? The misrepresentation of GOPs as variables exacerbates the analysis of multi-level phenomena (cf. Mackenzie 2006). If group and organizational behavior is processual and multi-level in nature, then the "shortcut" of using variables as substitutes for GOPs creates intractable and unnecessary problems in studying and testing group and organizational theories.

The present research practice of converting GOPs into tangentially related variables conceals the processual nature of the group and organizational phenomena. In so doing, the researcher inadvertently acts to change the subject and methods of one's research. In order to "get the science right," it seems practical and fruitful to develop a common representation of GOPs. To the extent to which these and the previous arguments are valid, an effort to develop a common representation for GOPs is worth the effort. The organization sciences would benefit if there truly was a common representation of GOPs which could create a standard to be used in research studies and in applications.

\subsection{An Example of a GOP Representation}

The discussion that follows in this article (and especially in the second) is necessarily abstract. It is easy to become lost in the formulations and derivations and lose sight of the basic idea of a GOP representation. A common sense example from everyday life that is generally familiar can provide an intuitive understanding of the basic idea and some of the attendant complexities of GOP representation. Consider the process of answering a telephone. This example of a simple GOP proceeds in three stages: (1) an individual answering a telephone; (2) the process of answering a telephone when there is a roommate, and (3) answering it with a telephone with "Caller I.D." capability and roommate. In the description of any GOP there are outcomes, considerations, and a means of linking the considerations to each other and to the outcomes. Answering a telephone is no exception.

Consider an individual with an old-fashioned land-line telephone in her residence. The telephone rings. What are the outcomes or responses? In the simplest case, there is a pair of outcomes: "I pick up" or "I don't pick up." Let's denote those outcomes by the vector $\mathbf{Y}_{1}$, where: $\mathbf{Y}_{1}=\left(\mathrm{y}_{1}, \mathrm{y}_{2}\right)=$ (I pick up, I don't pick up). In this simple case, there are two considerations: Is the phone ringing? And can I get to it in time? These considerations are denoted by the vector $\mathbf{C}_{1}$, where: $\mathbf{C}_{1}=\left(\mathrm{c}_{1}, \mathrm{c}_{2}\right)=$ (Is the phone ringing?, and Can I get to it in time?). The linkages between the elements of the considerations, $c_{1}$ and $c_{2}$, and the outcomes, $y_{1}$ and $y_{2}$, is illustrated in the graph, $\mathbf{F}_{1}$, or network of Figure 2. The linkages between the considerations and between the considerations and the outcomes are all binary, as indicated by the "Yes" and "No" answers. 


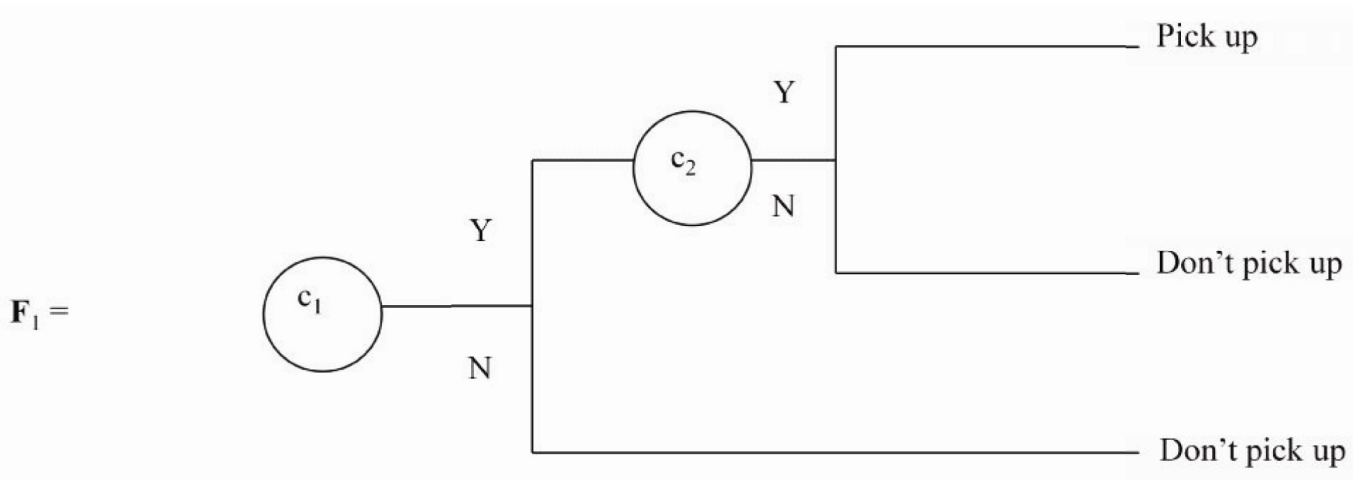

Figure 2. Process framework for answering a phone

So far, so good. But now let's add context by introducing a roommate. This gives us another outcome, $\mathrm{y}_{3}$, which is that the "Roommate picks up." This opens up two more considerations, $c_{3}$, "Is my roommate home?" and $\mathrm{c}_{4}$, "Can my roommate pick up?" The outcomes are: $\mathbf{Y}_{2}=\left(\mathrm{y}_{1}, \mathrm{y}_{2}, \mathrm{y}_{3}\right)=$ (I pick up, I don't pick up, roommate picks up) and $\mathbf{C}_{2}=\left(c_{1}, c_{2}, c_{3}, c_{4}\right)=$ (Is the phone ringing?, Can I get to it in time?, Is my roommate home?, Can my roommate pick up?) and the new graph or network, $\mathbf{F}_{2}$, is shown in Figure 3.

This could easily become more complex by adding information about the relationship between the roommates. For example, if one was jealous of the other, there could be new outcomes such as picking up and listening but not answering. Or, one may be avoiding certain callers and using the roommate to screen unwanted calls. The roommates might be business partners, where one acts as the receptionist in order to appear as a larger, more established enterprise.

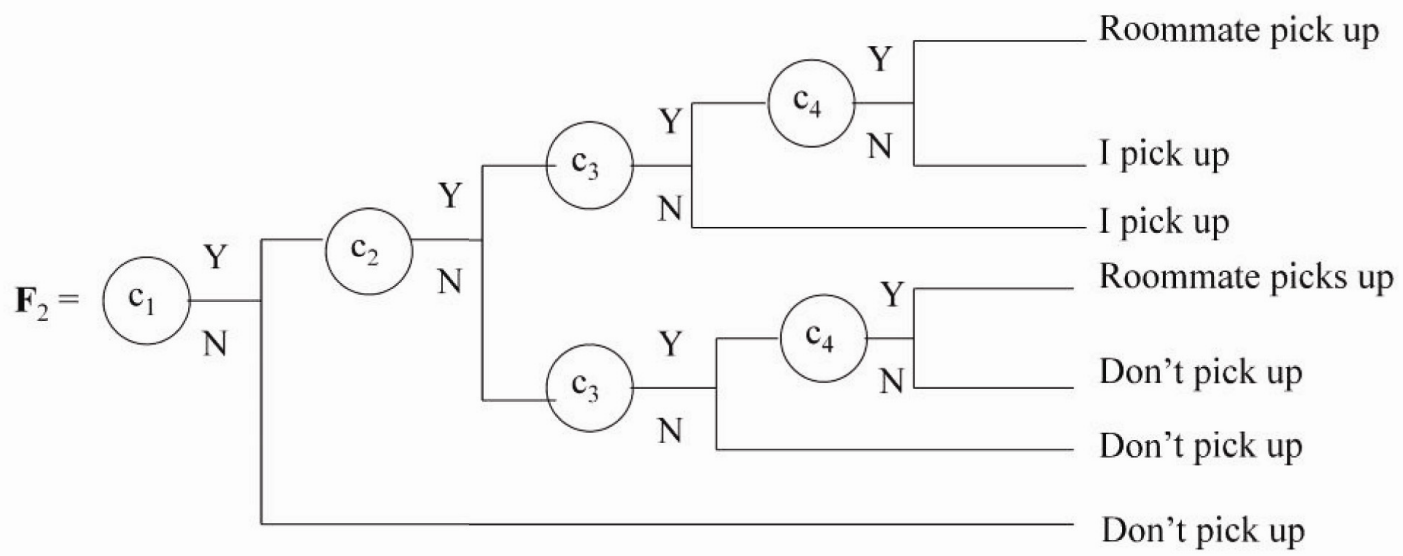

Figure 3. Process framework for answering a phone with a roommate

Of course, there are many technologies involved in telephones. Technology is part of the context, and technology affects the content of a GOP. Suppose, for example, that the phone has "Caller ID." This adds two new outcomes. The new set of outcomes, $\mathbf{Y}_{3}$, now has five possibilities: $\mathbf{Y}_{3}=\left(\mathrm{y}_{1}, \mathrm{y}_{2}, \mathrm{y}_{3}, \mathrm{y}_{4}, \mathrm{y}_{5}\right)=$ (I pick up, I don't pick up, roommate picks up, call back later, delete message) and two additional considerations. Thus, $\mathbf{C}_{3}$ becomes: $\left(\mathrm{c}_{1,}, \mathrm{c}_{2}\right.$, $\mathrm{c}_{3}, \mathrm{c}_{4}, \mathrm{c}_{5}, \mathrm{c}_{6}$ ) $=$ (Is the phone ringing?, Can I get to it in time?, Is my roommate home?, Can my roommate pick up?, Is the caller recognized?, Should the call be returned?) The new graph or network, $\mathbf{F}_{3}$, is illustrated in Figure 4. 


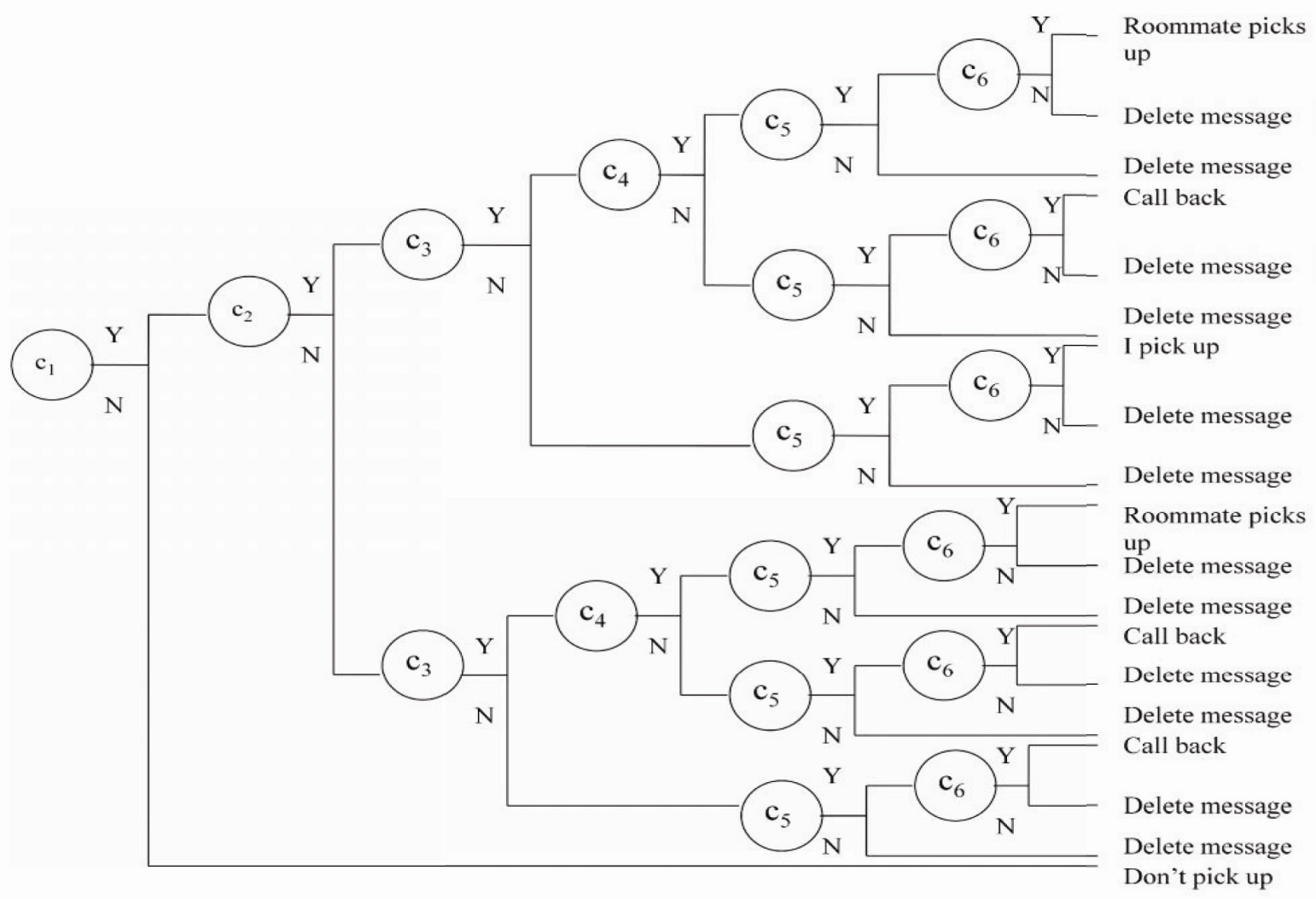

Figure 4. Process framework for answering a phone with a roommate and caller ID

These examples illustrate the capacity for even ordinary processes to become complex. In the case of answering a telephone, the examples barely scratch the surface. For example, Caller ID can include both the name and the number of the caller. The telephone call can be forwarded. There are the options of call waiting, text messaging, and call return services. The technology can include multiple lines, conference calls, virtual-team hookups, and even internet phone service. Moreover, on top of all this, there can be different structural relationships with the roommate, such as they are partners, master-servant, or married. The roommates could be estranged and the process of answering could be affected by physical disabilities, etc.

All three figures exhibit a sequence of considerations; each outcome is a path through the process framework from $\mathrm{c}_{1}$ to an outcome; there is equifinality in that different paths may yield the same outcome. Despite this, the three cases $\left[\left(\mathbf{Y}_{1}=\mathbf{F}_{1}\left(\mathbf{C}_{1}\right)\right.\right.$ then $\mathbf{Y}_{2}=\mathbf{F}_{2}\left(\mathbf{C}_{2}\right)$, and finally $\left.\mathbf{Y}_{3}=\mathbf{F}_{3}\left(\mathbf{C}_{3}\right)\right]$ exhibit the identical generic algebraic equation of $\mathbf{Y}=\mathbf{F}(\mathbf{C})$. This generic equation for describing group and organizational processes has had many applications and it is the basis for a common representation of GOP in the second article (Mackenzie, 2013).

\subsection{The Need to Consistently Represent Group and Organizational Processes}

There is a long tradition in mathematics of seeking common representations of classes of related mathematical problems. The resulting representation theorems allow a common formulation of a class of problems which facilitates and organizes development. Representation theorems facilitate mathematical research and make it more intelligible. Although the current study of group and organizational processes is far less rigorous than mathematics, there remains a tantalizing prospect that there may exist a common underlying representation of GOPs.

The pursuit of a common representation of group and organizational processes (GOPs) seems quixotic given the "messy" literature in the organization sciences and the large number of ad hoc descriptions of GOPs. The resulting buzz and confusion might seem too chaotic to even consider attempting to discover a common representation capable of producing useful results. How can one make sense of this utterly confusing variety of GOPs? GOPs can range from processes to amend an organization's culture to engaging in political attempts to change leadership, to negotiating and lobbying with government regulators, to generating accounting results, to 
processing purchase orders, and to allocating parking spaces. Langley (2009) documents the immense variety of GOPs and some of the research approaches to studying them.

Despite all of the distractions, there have been efforts to represent GOPs with analytical frameworks. These include, but are not limited to, the work of Mackenzie (2000, 2004, and 2009), Malone, et al (2003), Sowa (2000), and many using universal modeling languages, and process modeling in specialty areas such as quality assurance and logistics. The metaphor of a process to describe group and organizational phenomena is widespread and, in my opinion, a consensus is forming that group and organizational behaviors are inherently processual.

Of course it is one thing to represent a narrow class of GOPs that have evolved from special purposes (e.g., accounting control or inventory management) and a representation of GOPs that is common to a much wider class of GOPs. One can expect strongly entrenched theoretical and methodological versions of GOPs. Consequently, one should expect, once the intellectual conflict is joined, many competing models, representations, and intellectual posturing. It has proven fruitful in other areas of inquiry, such as decision theory, to erect an analytical framework for informing the debates. Laying bare the underlying definitions and axioms promotes comparison, contrast, and synthesis of competing approaches. These analyses foster the need for stated criteria and even desiderata for analyzing alternative models.

\subsection{Criteria for Feasible GOP Representations}

There are already several candidate GOP representations extant in the literature surrounding the science of group and organizational processes. Their rigor and approach is varied. If the science of group and organizational processes is to chose a common GOP representation, it would help to have stated criteria for defining the basic properties of a GOP representation. Any GOP representation that meets these is considered feasible. Ten desiderata are introduced in order to select from among the feasible GOP representations.

The primary criterion of a GOP representation is that it incorporates the breath of life. Because GOPs involve the actions and procedures of actual groups and organizations, they necessarily require a pair of properties. The first is the requirement of engaged agency by actual entities (called "Processual Agents" later in Mackenzie, 2013). That is, GOPs involve processual agents to enact and implement them. If there are no identified processual agents, then the proposed GOP representation is deficient. Second, GOPs involve the subject matter describing actual group and organizational behavior. A feasible GOP representation which cannot be directly linked to actual group and organizational processes is too abstract to be a feasible GOP representation. A candidate GOP representation that is not concerned with real group and organizational processes is defective. The subject matter of GOPs entails engaged processual agents involving actions undertaken by human groups and organizations.

The breath of life criterion requires an ontology of engaged processual agents acting in the context of groups and organizations. GOPs are not merely disembodied actions taking place in a vacuum. GOPs require engaged processual agents, events, considerations, and a structure for connecting the engaged processual agents to the steps or stages contained in the GOP.

An abstract, formal representation in the form of a computational procedure is not enough for a GOP. Of course, such formulations contribute to the how of a GOP, but absent the breath of life, they are deficient. Thus, while universal modeling language offer a powerful set of tools for assisting in the development of operational tools to formulate GOPs, they are not, by themselves, actual GOPs. Purely formal mathematical models of GOPs, tend to be tools and are not gifted by the breath of life. To go beyond the formal representations of processes, they need to incorporate the engaged processual agents, the subject of an actual group or organization doing something real, the place in which the processes unfold, and the needed resources and their characteristics-in-use.

There are four additional criteria to be met if a candidate GOP representation is to be a feasible one. After the first criteria of the breath of life, the other four are: (2) it offers a common algebraic structure to the representation of GOPs; (3) this algebraic structure also holds for specified classes of multi-level GOPs; (4) it is realizable in that it is capable of defining how actual groups and organizational processes can be deployed within the GOP representation; and (5) it is falsifiable.

The falsifiable criterion is especially important because the organization sciences could certainly benefit from an improved ability to identify and provisionally discard defective and deficient GOP representations. A GOP representation that meets these five criteria is feasible. But among the feasible some may be preferred to others. This leads to additional considerations called desiderata. These criteria and desiderata for a common GOP representation allow anyone to compare and contrast competing candidate GOP representations. 
It would not be surprising to eventually learn that like living creatures, there are different phyla of GOPs, each with special features. However, it is difficult to see how we can describe the phyla of GOP representations without first defining what we mean by the "animal" of which the phyla the GOP representations are included.

\subsection{Desiderata of a Common Representation of GOPS}

A GOP representation is also a technology for analyzing group and organizational processes. Any technology comes with some advantages and some costs. The nature of any technology is that it both reveals something different and hides what was before. For example, a sculptor working to create a marble statue uncovers the figure within but the end result masks the identity of the original block of marble. Today we have electronic devices that perform many marvels such as managing a community on Facebook, creating and sending manuscripts, creating videos, etc. We can fly armed drones great distances to report reconnaissance, take and transmit video pictures of what they see, lock on a target, and send a missile to destroy it. But, the identity of persons in the target area, what they are doing, the accuracy and reliability of those who gathered the intelligence for the mission, the purpose of the attack, and how the drone is designed, built, and managed are hidden. Newer technologies can provide benefits and promise by making older technologies either obsolete or less relevant. This revealing comes at the cost of hiding. Something is always lost. Without some agreed-upon standard, who knows whether a technology is, in fact, an improvement over an older one?

The study and analysis of group and organizational processes (GOPs) involves many possible technologies. These range from survey methods to participant-participation protocol analyses, to using unified modeling language tools. Each method involves one or more technologies that help one make use of the methods for studying and analyzing GOPs. But the results of these applications are no longer the actual group or organization being studied. The technologies can revive new insights about the GOPs but they also mask or hide the more complex reality: the wide variety of methods to study GOPs creates a need to choose among them.

The nature of any technology is that it is actually embedded and co-adapted with others. For example, an electric screwdriver requires electricity but the electricity requires transmission lines, electrical generators, etc. Technologies are specifically and purposefully adapted to other enabling technologies. But, in doing so, technologies both reveal and hide aspects. The compromises necessary to conform to their chosen enabling technologies force changes in the original concept.

Each technology of a GOP representation, like each blade of a Swiss army knife, has its own properties, advantages, and disadvantages. If each blade represents a concept and the enabling methods of a GOP representation, the Swiss army knife represents multiple concepts.

Each of these alternatives has its own specific properties and enabling technologies. But, given the plethora of GOP concepts, how can they be compared, constructed, and communicated?

The history of decision making may be appropriate here. Clearly, there are many methods, ranging from logic to complex mathematical programming tools. But, these methods, while highly diverse, can be analyzed by examining the basic underlying concepts. Different methods involve different definitions, assumptions, and axioms. The discipline of laying these bare for examination foster comparison and contrast, and illuminates opportunities for improvement and even elimination. This has not yet been done for GOP representations and it may be time to begin.

\section{Towards A Common Representation of GOPs}

There are many possible definitions of a group and organizational process. There are therefore many candidate representations of GOPs. As a starting point, it is useful to recognize that any choice of a GOP representation depends on the purposes and the conditions under which it is developed and applied.

One purpose of a GOP representation is the description of some classes of GOPs. These descriptions can occur at varying levels of realization. There are grand metaphors such as "life cycles," "seasons," and black-box diagrams popular in management texts (such as Figure 1). There are narratives that are not tied to any specific situation. Narratives are a type of storytelling. Narratives can act as powerful forms for communication and culture building in groups and organizations. But they are too abstract and "floating" to be fully realized GOP representations. There are unplaced and placed narratives. The concept of place is discussed in the second article of this series, "A working common representation of group and organizational processes" (Mackenzie, 2013). Metaphors, black boxes, and narratives lack realizations. They are still useful in presenting GOP concepts. A fully realizable GOP is very specific with well-defined outcomes, considerations, and an actual framework to connect them. The descent from grand metaphors all the way to fully realized GOPs is the path from vagueness to sharply focused reality. 
Another purpose of a GOP representation is proposing a theory of GOPs which uses a GOP representation to state the substantive theory. Such theories can vary widely in their realizability such as a theory of organizational strategy formulation in different market conditions and more specific theories of selected GOPs such as diversity management. Another purpose is to determine the means by which a GOP can be tested for validity and accuracy. In this case the method of hypothesis testing will involve constraints on the formulation of a GOP. If, for example, the means of testing involve use of mathematical statistics, then the GOP representation needs to define the means for converting the GOP process into numbers and the test must involve sufficient instances of the GOP in mind to allow sufficient degrees of freedom. On the other hand, it is also possible to represent each GOP to allow a pass/fail conclusion to test its statistical significance. A consideration in testing a GOP representation is whether or not one insists that it be refutable. Another purpose of a GOP representation is its use in consulting to improve or change existing processes such as managing the order-entry cycle. For each purpose there is the choice of the technology used to represent and apply the GOP representation.

However, it must be recognized that if the science of GOPs is to be vibrant, the four subcommunities need to be involved. The theorists need to work with the engineers, the managers tasked to apply it and the available tools that can be implemented. To go from a specific GOP representation to a common representation will encourage the GOP scientific community to work to improve the science. A common representation of GOPs would enable the four subcommunities of any GOP science to interact. Developments in any one can impact the work of the others more effectively if there is a common representation.

Basically, every candidate GOP representation entails definitions and axioms. If each candidate GOP representation is clear about its definitions and axioms, then the comparison and contrasts of rival GOP representations could be openly discussed. This brings us to consider criteria in the form of desirable features called desiderata.

There are at least ten possible desiderata for a common GOP representation. These are:

1. Its definitions and axioms are articulated. Different GOP representations entail different definitions and axioms. Laying them out for inspection would allow more effective analyses to compare, contrast, and synthesize them.

2. A GOP representation should provide an analytical framework. An analytical framework should allow for description of a GOP, specify how it could become realizable, and combine or change GOPs. An analytical framework stimulates methodological advances and allows deductions and syntheses.

3. A GOP representation needs to have the capacity for incorporating data. A GOP representation fails to provide a method for incorporating actual data lacks realizability and the ability to be tested for actual data. Furthermore, a GOP representation includes considerations which have their own set of relationships. Any GOP representation that cannot permit analysis of such relationships is sterile.

4. A common GOP representation can be used for multiple purposes. A GOP representation that is only applicable to a single purpose is not yet a common GOP representation.

5. The limitations of any GOP representation need to be defined so that one can determine the application to specific cases. For example, a representation of birth/death cycles may involve a system of difference equations and may not be applicable to more specific GOPs such as those for task completion. Knowledge of the limitations of a GOP representation provides boundaries for application and naturally stimulates attempts to find a means for overcoming them.

6. A common GOP representation should provide procedures for connecting with other GOPs as required. These connections include the capacity to "drill down" to open up more processual detail; aggregating constituent GOPs to higher levels; and the "chaining" of one GOP to another. An organizational design engagement to improve an organization will involve some very high level GOPs such as establishing the organization's strategic direction. But these high level GOPs need to be broken down into major functions of the organization, such as marketing, production, administration, etc. The marketing tasks can be further subdivided into sub task processes, etc.

7. A common GOP representation should be describable as the GOP in question and not confounded by assessments of various measures of the outcomes. For example, many GOPs do not directly involve money but are integral to the generation of profits and losses. Separating the GOP from its measures of outcomes simplifies the task of analysis. Given the plethora of possible measures, isolating the GOP itself from possible measures of its effectiveness and efficiency reduces complexity. A community bank in the USA 
has over 40 measures by which to analyze performance. For example, most GOPs within the bank are only indirectly connected to the ratio of assets and liabilities.

8. A common GOP representation ought to be testable. Is it working as designed? Is the theory correct? Can one create conditions under which the GOP can be evaluated for the accuracy of its predictions? A GOP representation that is testable is preferable to one that cannot be tested. More will be said about his problem in the second article.

9. A common GOP representation ought to have face validity. That is, once presented to others does it make sense? Is it understandable? Does it seem to capture the essence?

10. A common GOP representation generates applications that contribute value to the improvement of the GOP science. This tenth desiderata could be seen as the "so what" criterion. Probably the best way of considering the contribution of a GOP representation is to ask if there would be a loss if it were never proposed and whether it provides benefits to the science when it is proposed.

\section{Summary and Conclusions}

The concept of a science as a collection of interdependent communities of persons with a shared interest and commitment to the evolution of a body of knowledge opens up new avenues by which to explore how to improve the science of group and organizational processes. Figure 1 depicts a view of how its four subcommunities interact. The first subcommunity is comprised of the persons who are devoted to the evolution of substantive theories of GOPs. The second is those who work on the development of engineering methods to implement substantive GOP theories. The third subcommunity consists of the managers and consultants who work applying these deployable technologies for GOPs. The fourth group are those who work on developing deployable technologies for studying GOPs. Think of these subcommunities as the theorists, the engineers, the managers and consultants, and the tool-makers. These four subcommunities are interdependent, with actions and developments by anyone potentially affecting the others. Of course, these subcommunities do not always work in concert and their activities are not always directed to the development of the science of GOPs.

An examination of the relative progress of different scientific communities shows that some are far more effective than others in advancing their science. The science of group and organizational processes is not one of the rapidly advancing sciences. One reason for this is the relatively lower frequency of interaction among these subcommunities. The main thesis of the article is that this is due in part to the simple fact that this scientific community has not reached agreement on the basic concept of a GOP and, as a result, lack a common representation of them. This intellectual confusion hinders cooperation, obscures the meaning of advances, and makes it difficult to share new knowledge and to jettison approaches that do not seem to work. The result is a balkanization of the field into many competing "paradigms" and methods (cf. Lundberg and Young, 2005). The article goes on to suggest that now is the right time to begin to ask the question about how to define and represent GOPs.

Referring back to Figure 1, The Science of Group and Organizational Processes, a common GOP representation provides both a language and a technology for the science of group and organizational behavior. It helps formulate and test theories of group and organizational processes. It can stimulate the development of effective deployable tools. It can provide guidance to those developing the necessary engineering solutions to implement substantive theories of group and organizational processes. These substantive theories, supporting tools, and applicable engineering can also foster applications by managers and consultants who seek to improve and design group and organizational processes. In short, a common GOP representation will provide a boost to the science of group and organizational processes.

There are, of course, many problems to accomplishing the goal of developing a common definition and representation of group and organizational processes. And there are the questions about whether such efforts are either needed or useful. The article offers a number of arguments why we should begin to work out how to define and represent a basic and fundamental reality of group and organizational processes. This must be done in the face of very different issues for handling the enormous variety and complexity of GOPs. While the task is difficult, it is not impossible. It is time to begin.

This work is not being done in an intellectual vacuum. There already have been efforts to develop a definition of GOPs and there exist several suggested GOP representations. The evolution of a common concept and representation of GOPs is itself a process and one of the tasks will be to find the means to compare, contrast, and synthesize alternative common GOP representations. 
The first task toward this synthesis is to discuss criteria that a common GOP representation must meet. This article suggests these: (1) it offers a common algebraic structure that (2) holds for classes of multi-level GOPs, (3) is capable of incorporating actual group and organizational behavior, and (4) is falsifiable. In addition, candidate GOP representations can be compared using a set of desirable features or desiderata for such a common representation. There are ten such desiderata presented.

These desiderata include: (1) its definitions and axioms are articulated; (2) it should provide an analytical framework; (3) it is capable of incorporating actual data; (4) it can be used for multiple purposes; (5) it has definable limitations; (6) it allows for linking with other GOPs; (7) it should separate the GOP itself from evaluations of it; (8) it should be testable; (9) it should have face validity; and (10) it provides value to the science.

Establishing a common GOP representation is a necessary but not sufficient condition for improving the science of group and organizational processes. It is necessary because GOP theory development, tool making, engineering, and application involve GOP processes. Having a common language facilitates communication and productive interdependencies within and among the four subcommunities. It is a necessary early step which provides a firmer foundation for the evolution of this science. It is expected that the common GOP representation will undergo changes as the science of group and organizational processes evolves. Having criteria and desiderata for comparing and contrasting and synthesizing GOP representations helps to frame these debates and controversies.

\section{Acknowledgements}

Thanks to Craig C. Lundberg (NT), Donald F. Utter, and Susan M. Treber for their constructive suggestions for improving this article. Thanks are also owed to reviewers whose criticisms on the manuscript led to improvements.

\section{References}

Avital, M. (2000). Dealing with time in social inquiry: A tension between method and lived experience. Organization science, 11(6), 665-673.

Bogost, I. (2006). Unit operations: An approach to videogame criticism. Cambridge, MA: MIT Press.

Crick, F. H. C., \& Watson, J. D. (1953). Molecular structure and nucleic acids: A structure for deoxyribonucteic acid. Nature, 171, 737-738.

Cyert, R. M., \& March, J. M. (1963). A behavioral theory of the firm. New York: Prentice-Hall.

Greenwood, R., \& Hinings, C. R. (1996). Understanding radical change: bringing together the old and new institutionalism. Academy of Management Review, 21(4), 1022-1054.

Harmon, G. (2009). Prince of Networks: Bruno Latour and metaphysics (Anamnesis). Melbourne, Australia: Repress.

Kilmann, R. H. (2001). Quantum organizations: A new paradigm for achieving organizational successes and personal meaning. Palo Alto, CA: Davies-Black Publishing.

Langley, A. (1999). Strategies for theorizing from process data. Academy of Management Review, 24(4), 691-710.

Langley, A. (2009). Studying processes in and around organizations. In D. Buchanan, A. Bryson, (Eds.), Sage Handbook of Organizational Research Methods, 24, 409-429.

Lundberg, C. C., \& Young, C. A. (2005). Foundations of inquiry: Choices for tradeoffs in the organization sciences. Stanford, CA: Stanford Business Books.

MacIntyre, A. (1984). After virtue: A study of moral theory (2nd ed.). Notre Dame, IN: University of Notre Dame Press.

Mackenzie, K. D. (1976a). A theory of group structures (Vol. I: Basic theory). New York: Gordon and Breach Science Publishers, Inc.

Mackenzie, K. D. (1976b). A theory of group structures (Vol. II: Empirical tests). New York: Gordon and Breach Science Publishers, Inc.

Mackenzie, K. D. (1986). Organizational design: The organizational audit and analysis technology. Norwood, NJ: Ablex Publishing Corporation.

Mackenzie, K. D. (1994). The science of an organization, Part 1: A new model of organizational learning. Human Systems Management, 13, 249-258. 
Mackenzie, K. D. (2000). Processes and their frameworks. Management science, 46(1), 110-125.

Mackenzie, K. D. (2004). The process approach to multi-level organizational behavior. In F. Yammarino, \& F. Dansereau (eds.), Research in multi-level issues: Multi-level issues in organizational behavior and processes (Vol. 3, pp. 347-417). Oxford, UK: Elsevier Science.

Mackenzie, K. D. (2006). Some ideas about testing processual theories. In F. Yammarino, \& F. Dansereau (Eds.), Research in multi-level issues: Multi-level issues in social systems (Vol. 5, pp. 443-475). Oxford, UK: Elsevier Science.

Mackenzie, K. D. (2007). The god of variance has feet partly of iron and partly of baked clay. International Journal of Organizational Analysis, 15(1), 5-22.

Mackenzie, K. D. (2009). Process skeletons and functions. Human Systems Management, 28(4), 201-212.

Mackenzie, K. D. (2013). A working common representation of group and organizational processes. Engineering Management Research, 2(1).

Malone, T. W., Crowston, K., \& Hermann, G. A., (Eds.) (2003). Organizing business knowledge: The MIT process handbook. Cambridge, MA: MIT Press.

Melcher, A. J. (2012). Formulating a research question: Considerations and guidelines. Unpublished manuscript. Southern Illinois University at Carbondale.

Mohr, L. (1982). Explaining organizational behavior: The limits and possibilities of theory and research. San Francisco, CA: Jossey-Bass.

Numagami, T. (1998). The infeasibility of invariant laws in management studies: a reflective dialogue in defense of case studies. Organization Science, 9(1), 2-15.

Orlikowski, W. J. (1996). Improvising organizational transformation over time: a situational change perspective. Information Systems Research, 7(1), 63-92.

Pettigrew, A. M. (1985). The awakening giant: Continuity and change on ICI. Oxford, UK: Blackwell.

Pettigrew, A. M. (1997). The double hurdles for management research. In T. Clarke (ed.), Advancement in organizational behavior: Essays in honour of D. S. Pugh (pp. 277-296). London, UK: Dartmouth Press.

Pettigrew, A. M., \& Whipp, R. (1991). Managing change for competitive success. Oxford, UK: Blackwell.

Pettigrew, A. M., Woodman, R. W., \& Cameron, K. S. (2001). Studying organizational change and development: challenges for future research. Academy of Management Journal, 44(4), 697-713.

Rescher, N. (1996). Process metaphysics: An introduction to process philosophy. Albany, NY: State University of New York Press.

Sowa, J. F. (2000). Knowledge representation: Logical, philosophical, and computation foundations. Pacific Grove, CA: Brooks/Cole.

Van de Ven, A. H., \& Huber, G. P. (1990). Longitudinal field research methods for studying processes of organizational change. Organization Science, 1(3), 213-219.

Van de Ven, A. H., Angle, H. L., \& Poole, M. S. (1989). Research on the management of Innovation: The Minnesota Studies. New York, NY: Ballinger/Harper and Row.

Weick, K. (1969). The Social Psychology of Organizing. Reading, MA: Addison-Wesley. 